\title{
JSSHP Board Members and Councilors 2018-2019
}

\section{Executive Board Members:}

Hiroyuki Seki (Chairperson of the Executive Board)

Atsuhiro Ichihara (Vice Chairperson of the Executive Board, Chairperson of International Relations Committee)

Tadashi Kimura (Chairperson of General Affairs Committee)

Kenjiro Takagi (Chairperson of Scientific Program Committee)

Mineo Yamazaki (Editorial Committee)

Yusuke Suzuki (Editorial Committee)

Tomoaki Ikeda(Chairperson of Public Relations Committee)

Akihiko Sekizawa (Treasurer)

Shigeru Saito (Chairperson of ISSHP 2020 Nara Organizing Committee)

\section{Auditors and Supervisors:}

Satoru Takeda, Tatsuo Yamamoto

\section{Board Members:}

Tomoko Adachi, Atsuo Itakura, Horoaki Itoh, Kazuhiko Ino, Shinya Unno, Hideaki Ohkuchi, Yasumasa Ohno, Masaki Ogawa, Hidetaka Katabuchi, Kiyoko Kato, Keizo Kanasaki, Naohiro Kanayama, Yoshimasa Kamei, Yoshiki Kudo, Masayasu Koyama, Hiroshi Sameshima, Koichiro Shimoya, Junichi Sugawara, Mayumi Sugiura, Motoi Sugimura, Takashi Sugiyama, Yoshikatsu Suzuki, Kazuo Sengoku, Koichiro Takagi, Toshiyuki Takeshita, Kanji Tanaka, Mamoru Tanaka, Kiyomi Tsukimori, Katsuo Terui, Yukihiro Terada, Akihito Nakai, Osamu Nakamoto, Takeshi Nagamatsu, Hisashi Narahara, Katsuhiko Naruse, Akihiro Nawa, Tomoyoshi Nohira, Toshiyuki Hata, Akiyoshi Fukamizu, Tomoyuki Fujii, Keiya Fujimori, Kenichi Furuya, Yoshio Matsuda, Keiichi Matsubara, Shigeki Matsubara, Nobuaki Mitsuda, Naoyuki Miyasaka, Atsuko Murashima, Hirohito Metoki, Hideto Yamada, Jun Yoshimatsu, Akihiko Wakatsuki, Kazushi Watanabe

\section{Secretary Members:}

Shintaro Makino (Secretaly General),

Ikuno Kawabata (Vice Secretary General), Kazuya Mimura (Vice Secretary General),

Takayuki Iriyama, Takafumi Ushida, Kaiko Koide, Eiji Kondo, Nobuyuki Takahashi, Jun Takeda, Akitoshi Nakajima, Yoshihiro Hidaka, Shigetaka Matsunaga, Mamoru Morikawa

\section{Councilors:}

Yukio Iida, Ishii Keisuke, Takayuki Iriyama, Takafumi Ushida, Takashi Oba, Kazuhide Ogita, Hideki Kameya, Ikuno Kawabata, Masato Kamitomo, Nobuyuki Kita, Takashi Kitanaka, Tomoyuki Kuwata, Keiko Koide, Kaori Koga, Gen Kobashi, Eiji Kondo, Masatoshi Sakai, Koichi Shinohara, Takao Sekiya, Yoshikazu Takakuwa, Nobuyuki Takahashi, Hidenori Takahashi, Jun Takeda, Yoshiharu Takeda, Daisuke Tachibana, Fumihisa Chishima, Hajime Tsunoda, Yuichiro Nakai, Akitoshi Nakajima, Masahiko Nakata, Mikiya Nakatsuka, Hiroaki Nakamura, Kazuo Hashiguchi, Nobuhiro Hidaka, Hiromi Hamada, Rijin Fukui, Hiroshi Fukuda, Toru Funakoshi, Seishi Furukawa, Kazutoshi Maeda, Shintaro Makino, Naoki Masaoka, Hideo Matsuda, Shigetaka Matsunaga, Kei Miyakoshi, Mitsui Muraoka, Kazuya Mimura, Hidehiko Miyake, Isamu Murakami, Mamoru Morikawa, Hiroki Morita, Kenji Yamaguchi, Shun Yamada, Atsushi Yoshida, Jun Yoshida, Takahiro Yamashita, Tomoo Waseda 


\section{Congress President}

Shigeru Saito (University of Toyama)

ISSHP 2020

to be held in Nara, October 14-17, 2020

\section{Tadashi Kimura (Osaka University)}

The $39^{\text {th }}$ Annual Congress of the Japan Society for the Study of Hypertension in Pregnancy to be held in Osaka, November 2-3, 2018

Kenjiro Takagi (Jichi Medical University Saitama Medical Center)

The $40^{\text {th }}$ Annual Congress of the Japan Society for the Study of Hypertension in Pregnancy to be held in Saitama, 2019 